\title{
A Web Platform and a Decision Model for Computer-Interpretable Guidelines
}

\author{
Tiago Oliveira, Paulo Novais, and José Neves \\ University of Minho, Braga, Portugal \\ \{toliveira, pjon, jneves\}@uminho.pt
}

\begin{abstract}
Situations of medical error and defensive medicine are common in healthcare environments and have repercussions in the quality of care under offer. The occurrence of adverse events and the increase of healthcare expenses are some of the consequences of medical malpractice. Indeed, these situations may be prevented by encouraging the compliance with Clinical Guidelines (CGs). However, the current format of CGs proved to be disadvantageous for real-time application, i.e., they may not provide recommendations to healthcare professionals when required, and on time. The introduction of Computer-Interpretable Guidelines (CIGs) may provide a solution to this problem, however they are not widely implemented and there are some issues that need to be contemplated. Indeed, in this paper it is presented the CompGuide project for guideline representation and sharing, combined with the handling of incomplete information in that context.
\end{abstract}

\section{Introduction}

Clinical Guidelines (CGs) [1] are documents based on scientific evidence and consensus among experts that provide recommendations to deal with specific clinical cases. Their main objective is to structure the tasks of a clinical process according to the health condition of a patient.

Official development programs of CGs started between the late 70s and the early 80 s. Since then, CGs have progressed significantly. The medical community has continuously addressed the weaknesses of CGs and their development. Initially they were solely based on informal consensus among clinical experts, working on a regional base, and the guideline development group was exclusively composed by physicians. Now guidelines are based on rigorous scientific evidence evaluated by multidisciplinary groups of professionals, including a wide range of expertise from different scientific fields, namely from management of human resources to exam pactice. In fact, the need for a standardized evidence grading system led to the creation, in the year 2000, of the Grading of Recommendations Assessment, Development and Evaluation (GRADE) [2] project, an initiative for evidence grading. The guideline development programs evolved to national development programs and have spread across the world with the help of the Guidelines International Network (G-I-N) [3], created in 2002 and with a membership of 85 organizations from 43 countries. 
Development of CGs is a central subject in the medical community, since increased compliance with standards may provide a solution to mitigate the effects of medical errors and defensive medicine. Medical errors are mistakes committed by healthcare professionals that result in harm to the patient. It is a universal problem, namely in the United States (US), as it is shown in the bar graph of Fig. 1, but also in Europe. Studies show that the rate of adverse outcomes in London hospitals is $10.8 \%$ and the fatality rate resulting from medical error is $8 \%$ [4]. These numbers may not seem too adverse, but from the perspective of patients that put their lives on the hands of healthcare professionals, they are quite expressive and object of concern.

On the other hand, defensive medicine occurs when a healthcare professional avoids treating certain patients or orders treatments and exams to avoid criticism and eventual lawsuits. Studies about defensive medecine in breast cancer detection estimate that nearly $50 \%$ of the tested women will receive a false positive [6]. This puts them in emotional distress and may create a state of pseudo-disease. In some cases, the trust in their physician may be seriously undermined. The effectiveness of CGs in addressing these issues can be seen in a case study for ischemic heart disease, in which an increase of $10 \%$ in guideline compliance was associated with a decrease in $10 \%$ in mortality [7].

CGs have come a long way since they were idealized. However there are still some issues to be addressed in order to be able to effectively mitigate medical errors and defensive medicine. In the following sections we will point the shortcomings of paper-based CGs as well as related work in the field. In the last section we will present the CompGuide project, which is aimed at the representation of clinical knowledge contained in CGs, increasing their availability and the handling of incomplete information in the clinical process [1]. Soft computing techniques in the form of clinical decision support systems have been used by researchers in this field to manage the issue of incomplete information in the clinical process [9].

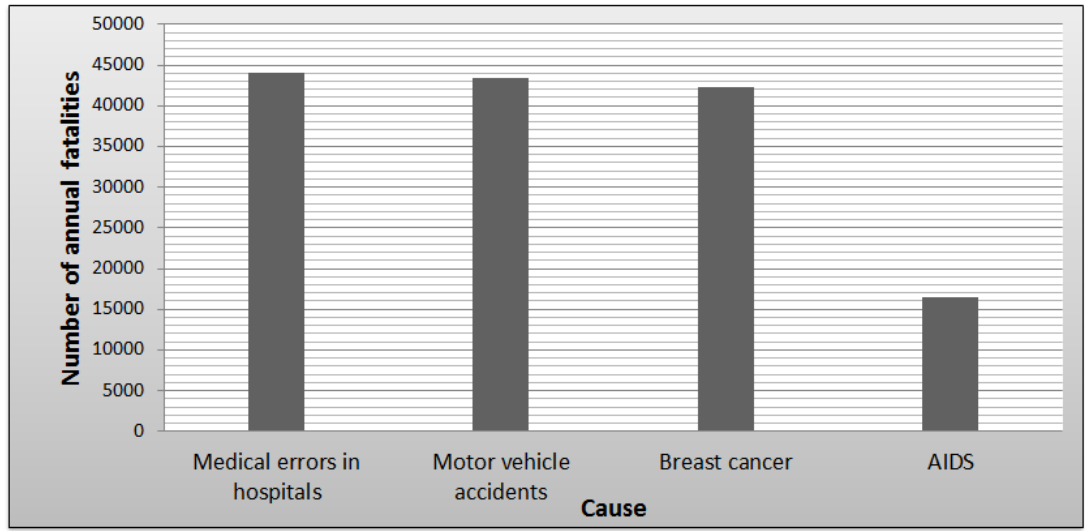

Fig. 1. Bar graph showing the annual fatalities in the US caused by medical errors in hospitals, motor vehicle accidents, breast cancer and AIDS [5]. 


\section{Shortcomings of Paper-based Clinical Guidelines}

There are some issues with the text format of CGs, pointing out that long textual documents are difficult to consult. The information takes too long to be obtained and the texts are susceptible to ambiguous interpretations, given the lack of accuracy of the medical concepts that are used and their unstructured nature [8]. To a healthcare professional that has to enforce and perfect his/her clinical practice, it is nearly impossible to collect, assess and interpret the recommendations of these CGs at the moment of the delivery of care. The maintenance of this type of documents is problematic, since a modification usually implies restructuring the whole document. This is the reason why most guidelines are only revised, thus making it difficult to keep up with the rapid development of scientific knowledge.

Healthcare professionals are also concerned that compliance with CGs may lead to an inflexible clinical practice, too focused on rules to follow or respect. They fear that this will restrain their ability to adjust medical procedures to the context under which they are inserted, by reducing their decision-making capability. Justified variability in clinical practice is necessary when there is the need to accommodate differences in healthcare systems, in the characteristics of the populations (e.g., social, demographic, cultural, health condition) or when the patient and the healthcare professionals have preferences among clinical procedures that are logically acceptable [8].

From the analysis of the current limitations of CGs, it is evident that they should develop an interaction with the user, which in this case is the healthcare professional. Guidelines should provide real time recommendations as the clinical process unfolds, taking into account the state of the patient, and the preferences of the healthcare professionals in control. In order to facilitate their update they should present a modular structure, so that only a portion of the guideline may be adjusted without revising the whole document. These perceptions of interactivity and modularity are essential for the creation of living guidelines, the next stage in the evolution of CGs.

\section{Computer-Interpretable Guidelines}

CIGs are representations of CGs in a digital format. A CIG system is essentially a Clinical Decision Support System that integrates some basic features, namely a guideline depiction model and an execution engine [10]. Trying to stay up-todate, in this review we will mention the current trends in the development of CIGs and afterwards the insight mechanims that enhance this field.

\subsection{Current Trends}

Currently there are few CIG systems available. However we will address them by their depiction models and mention the execution engines available for each one. 
The depiction models present in this review are Arden Syntax [11], Guideline Interchange Format (GLIF) [12], PROforma [13] and SAGE [14].

Arden Syntax was developed in 1989 and is now a standard of Health Level 7 (HL7) [11]. The current version of Arden Syntax is Arden Syntax 2.8. This approach focuses on sharing simple and independent guidelines as modules. Each CG is modeled as a Medical Logic Module (MLM), which comprises relevant knowledge.

GLIF represents an effort of Intermed Collaboratory in the development of a sharable CG representation model [12]. The GLIF depiction model dates from 1998 and its current version is GLIF3. It consists of a set of five classes, each one representing a step in the clinical process. This approach is task-based and follows the Task Network Model (TNM), so every moment of the clinical process is labeled as a Decision Step, Patient State Step, Branch Step, Synchronization Step or Action Step. There is not a formal method of representation of temporal constraints between steps in GLIF, however this is assured by a subset of Asbru temporal language, which is the strong argument of this approach. At Columbia University, GLIF is being integrated with the Clinical Event Monitor and the Computerized Physician Order Entry (CPOE) system to provide clinical decision support. The GLIF3 Guideline Execution Engine (GLEE) is a tool for executing guidelines in this format.

In 1998, the Advanced Computation Laboratory of Cancer Research of the United Kingdom initiated the development and assessment of the PROforma depiction model [13]. The objective of this model was the construction of guidelines as flowcharts where the nodes are instances of pre-defined classes of tasks. The classes are Plans, Actions, Decisions and Questions. Each class has a set of attributes that reflects its information needs. Among the execution engines for PROforma, Arezzo and HeCaSe2 are to be highlighted [13].

The SAGE (Standards-Based Sharable Active Guideline Environment) project is a collaboration of six research groups (IDX Systems, University of Nebraska Medical Center, Intermountain Health Care, Apelon Inc., Stanford Medical Informatics and the Mayo Clinic) [14]. SAGE includes a guideline depiction model and a guideline execution engine. Its objective is to establish an infrastructure to enable sharing guidelines in heterogeneous clinical information systems. SAGE is involved with organizations of healthcare standards (mainly HL7) to bridge the gap between guideline logic and real life implementations, and it is considered the evolutionary successor to EON and GLIF. The SAGE depiction model for Clinical Guidelines consists of Guideline Recommendation Sets, which are composed as a graph of Context Nodes. These Context Nodes can be Action Nodes, Decision Nodes and Routing Nodes. The patient state is retrieved directly from the electronic health record of the healthcare entity. SAGE makes use of terminologies and ontologies such as SNOMED-CT and LOINC. However SAGE is a relatively recent approach and shows some deficiencies concerning the integration of standards. 
Recently, new approaches for guideline modeling, aimed to improve the aspects of the previously mentioned ones, are emerging, of which the Guideline Acquisition, Representation and Execution (GLARE) [15] is to be noticed.

\subsection{Development Perspectives}

From the study of the different CIG systems, it is possible to extract some common features that should be in mind in the development of a CIG system, namely:

- a guideline repository with different versions of guidelines;

- a guideline editor that enables the acquisition of new guidelines;

- a guideline representation language with a set of primitives of the tasks of the clinical process;

- access to the Electronic Medical Record (EMR) and to a Clinical Management System (CMS); and

- use of terminology and information standards.

It is common sense to state that a paper format cannot be compared to a computerized guideline, since the first cannot be processed electronically. But, our perspective is how an electronic format can be more advantageous and provide a new set of tools to facilitate the work of healthcare professionals. From this point of view, besides addressing the drawbacks of the paper format, CIGs may have a positive impact in the development process of guidelines. The computerized format and an underlying development framework enable the implementation of features for collecting evidence and to grade them, as well as for group decision making. The application of formal methods, based on Mathematical Logic [19, ?] may be used to structure the development process and thus to prevent the elaboration of weak guidelines. Although these are not the main goals of the present work, they are interesting possibilities brought by electronic guidelines.

We have also identified two aspects where CIG systems are lacking. A good feature that could be implemented in these systems is a web-based version of the guideline editor, thus enabling healthcare professionals to freely build their guidelines online. This would be advantageous since it would allow the development of collaborative features of guideline development among different clinical experts scattered across a wide geographical area. It would solve one of the major problems of guideline distribution, which is the choice of the most suitable mean to deliver these recommendations to care workers.

Another aspect is the handling of incomplete information that occurs in the clinical process and the impact it has in decision making. Cases of uncertainty, inexactitude and incoherence in the clinical process may stop the flow of information from the observation phase to the decision phase, thus preventing a healthcare professional from devising a suitable treatment plan for a patient.

\section{The CompGuide Project}

The CompGuide Project is an initiative with the following objectives: 
- the development of a web platform for acquisition and execution of CGs in a digital format;

- the development of a new guideline depiction model that captures all the information needs of the clinical process; and

- the development of a clinical decision model that combines guideline recommendations with incomplete information that may arise from the clinical process.

In the following sections we will address the different components of this project, like features, and discuss how they may be implemented, with some detail.

\subsection{Architecture of the Web Application}

Web applications are the ideal support for delivering and gathering information. They are platform independent and are available at any time at any local access. This ensures that our purpose covers a wide range of devices, enabling people who want to collaborate to share their work and vision. The application uses the JavaServer Pages (JSP) [16] technology to add dynamic content to html pages. The architecture of the application is displayed in Fig. 2. It follows the Model-View-Controller (MVC) design pattern, where there is a separation between request handling, business logic and interface. Under this model, a servlet handles all the requests, manages the logic and instantiates the Java beans. The Java beans contain the Guideline Constructor and the Guideline Inference Engine, which have access to the Data Sources, i.e., a database containing data about the patient, a repository of guidelines in a MySQL database, the Unified Medical Language System (UMLS) and Terminology Services (UTS) [17]. The UMLS integrates and distributes key terminology and has three knowledge sources: the Metathesaurus that maps medical terms synonymous of the same medical concept (e.g., SNOMED CT, LOINC), the Semantic Network that establishes associative connections between terms (e.g., cause and effect) and the SPECIALIST lexicon, for syntactical, morphological and orthographic analysis of the terms. The connection to the UTS is possible through a Java API provided by UMLS. Finally, the JSP obtains the response from the beans and formats the response accordingly.

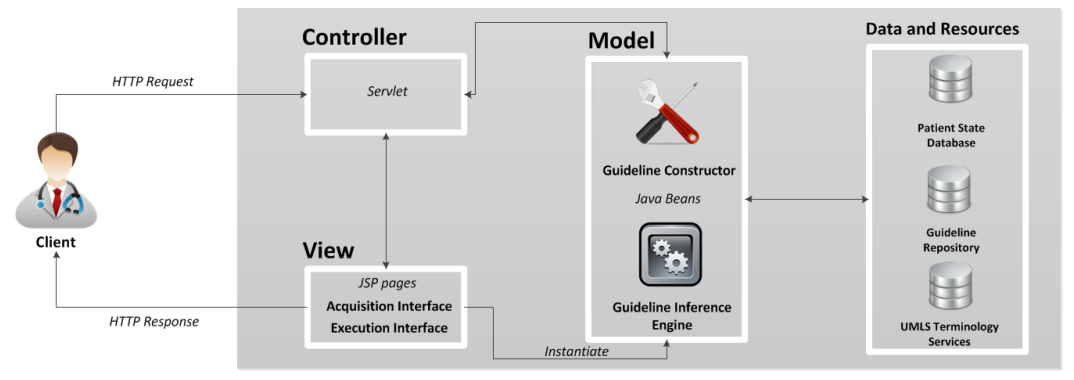

Fig. 2. The architecture of the CompGuide web application. 


\subsection{Guideline Representation}

Since there is not a standard model for guideline representation, we intend to develop a depiction model capable of performing that task, that is integrated with a standard terminology of clinical terms, the UMLS, and is in accordance with standard models of clinical information, such as the HL7. This will establish the necessary infrastructure to deal with interoperability issues with applications that are already used in clinical settings. The approach to guideline modeling of CompGuide presents an abstract view of decision making processes and task management during a clinical action or process [18]. The model is depicted in Fig. 3. A CG is viewed as a set of tasks, to which is given the designation of plan.

A plan contains instances of primitive classes that reflect the assignments of a CG. An action is an undertaking that represents a clinical procedure to be performed by the healthcare professional. To feed inputs to the system we use the question task. When a decision point is reached in the guideline workflow, it is used the decision task, which contains rules that associate conclusions to the parameters and values of the state of a patient. Action, question and decision are the atomic tasks of the model. It was considered that any type of atomic task gravitates around a clinical term, either it designates a parameter of the patient state, a clinical procedure or a clinical exam. The scheduling constraints are defined by attributes such as previous and next, that contain the id(entification) of the tasks that come before and after the present duty.

The other types of tasks defined in a plan are aimed at controlling special cases of the clinical workflow. The aggregation module groups tasks that are part of a cycle or iteration, creating the conditions for the user to define their periodicity, duration and objective. It is also used to represent tasks that belong to alternative pathways of the clinical workflow, like the ones that follow a decision task, in which the system chooses the next undertaking of the clinical process according to the conclusion reached at the decision step. The aggregation module can also group simultaneous tasks.

Another relevant aspect of the model is the terminology subclass of plan. Terminology comprehends the terms used in all the tasks of the plan along with their Concept Unique Identifier (CUI), which is a code used in the UMLS Metathesaurus to identity a concept and associate the different terms that may be used as a synonymous. 


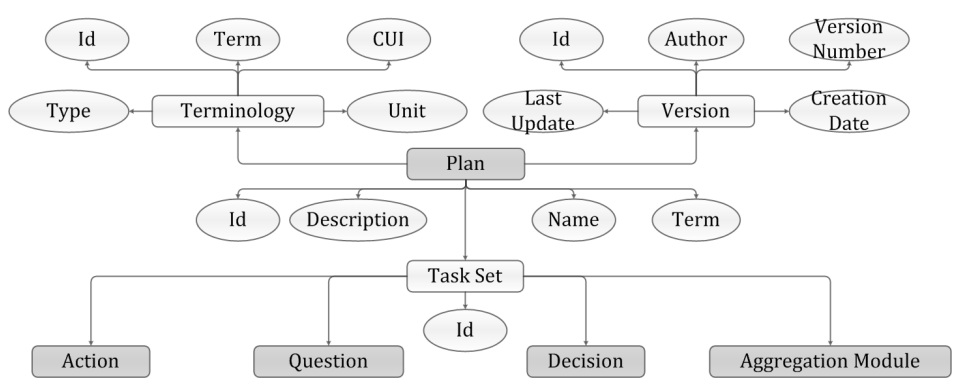

Fig. 3. The CompGuide guideline model and its different types of tasks.

\subsection{Clinical Decision Model}

Before applying a clinical decision model that includes incomplete information, it is necessary to represent it in an appropriate way. Extensions to the Language of Logic Programming (ELP) $[19,20]$ is one of the few techniques that enable this representation, using Mathematical Logic. ELP uses negation-byfailure and classic negation to represent explicit negative information. From this point of view the absence of information is also taken into account in the decision model. ELP enables the representation of cases of incomplete information about the state of a patient. For instance, in cases of inexactitude where there are different possibilities for the value of a clinical parameter, these possibilities are represented as abducibles or exceptions. In cases of uncertainty, if the value of the clinical parameter is unknown, this is represented as a null value.

The example of Fig. 4 is a simplified fragment of the ATP III guideline for Detection and Treatment of High Blood Cholesterol in Adults (developed by the US National Heart, Lung and Blood Institute) that is responsible for the detection of metabolic syndrome (ms). The information about the patient John is a typical case of incomplete information.

Decision making in these situations requires the use of an information quantification method. The Quality-of-Information (QoI) [19,?] is a methodology associated with ELP. It is defined in terms of truth values taken in the interval $[0,1]$ that are attributed to the clinical parameters of the patient according to their number of abducibles and null values. Given this, it is possible to calculate the QoI of each condition in a decision and calculate scores for each conclusion with the relative weights of its conditions. The decision model of CompGuide is based upon ELP and QoI [18]. The first stage of the decision model is the Formulation of Hypotheses where it is carried out a survey of the available options in terms of a decision. The following stage is Voting, which includes the Evaluation of Conditions and the Evaluation of the QoI. The scores of the options are calculated and, in the Clinical Rule Selection, the option with the best score is selected. In the Clinical Task Selection, the next task in the clinical workflow is selected through a matching with its trigger condition. 


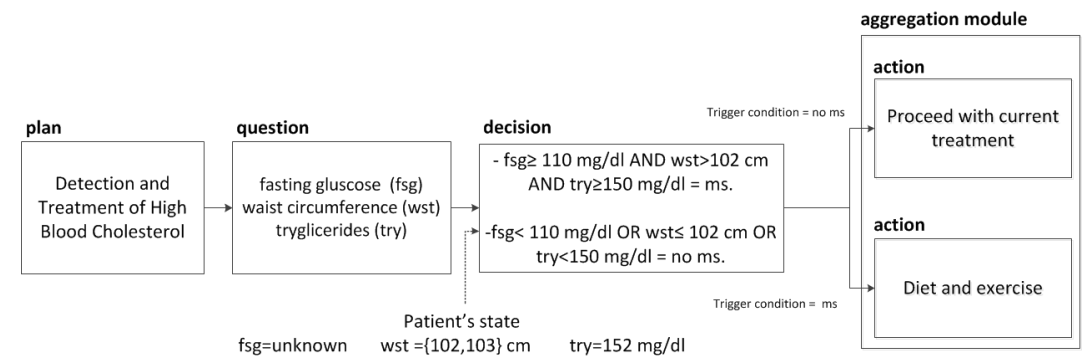

Fig. 4. Fragment of the guideline for Detection and Treatment of High Blood Cholesterol in Adults.

\section{Conclusions and Future Work}

The proposed system gathers the main features of the available CIG systems and goes beyond by trying to develop a web collaborative platform. The work requires further development of the web application and continuous improvement of the guideline model and the decision model. As observed the guideline model should be expressive enough to allow the definition of the different types of tasks that compose a guideline, as well as scheduling constraints on those tasks, without increasing the complexity of the model, in order to allow an intuitive acquisition of guidelines in the web application. The QoI approach really enhances our methodology for problem solving, since it offers a way to deal with incomplete information concerning the cases of missing information and conflicting/redundant and contradictory information. Indeed, uncertainty is dealt with in terms of the causality between symptoms and diseases, measured by the different scenarios that model and drive the universe of discourse.

What this approach offers is an intuitive environment for building and executing guidelines, through an expressive model, accessible to any healthcare professional and patient, as well as a decision model capable of processing incomplete and uncertain information. These features will offer a solution to current problems of guideline development, which none of the existent approaches managed to address.

\section{Acknowledgements}

This work is funded by National Funds through the FCT - Fundação para a Ciência e a Tecnologia (Portuguese Foundation for Science and Technology) within project PEst-OE/EEI/UI0752/2011". The work of Tiago Oliveira is also supported by a doctoral grant by CCTC - Computer Science and Technology Center (UMINHO/BI/004/2012).

\section{References}

1. Rosenbrand K, Croonenborg J, Wittenberg J (2008) Guideline Development. In Teije A, Miksch S, Lucas P (eds) Computer-based Medical Guidelines and Protocols: A Primer and Current Trends, pp. 3-22. 
2. Kavanagh B (2009) The GRADE System for Rating Clinical Guidelines. PLoS Medicine vol. 6, pp. 5.

3. Ollenschlger G (2003) International Guideline Network G-I-N (Guidelines International Network): Background and goals. Medizinische Klinik Munich Germany 1983 vol. 98 , no. 7 , pp. 411-412.

4. Kalra J (2004) Medical errors: an introduction to concepts. Clinical biochemistry, vol. 37 , no. 12 , pp. 1043-51.

5. Brennan T (2000) The Institute of Medicine report on medical errors-could it do harm? The New England Journal of Medicine, vol. 96, no. 6, pp. 1123-1125.

6. Chawla A, Gunderman R (2008) Defensive medicine: prevalence, implications, and recommendations. Academic radiology, vol. 15, no. 7, pp. 948-9.

7. Asher E, Parag Y, Zeller L, Yerushalmi R, Reuveni H (2007) Unconscious defensive medicine: The case of erythrocyte sedimentation rate. European journal of internal medicine, vol. 18 , no. 1 , pp. 35-8.

8. Woolf S, Grol R, Hutchinson A, Eccles M, Grimshaw J (1999) Potential benefits, limitations, and harms of clinical guidelines. British Medical Journal, vol. 318, no. 7182, pp. 527-530.

9. Abraham A (2009) Hybrid Soft Computing and Applications. International Journal of Computational Intelligence and Applications, World Scientific Press, Singapore, vol. 8, No. 1, 2009, pp. 5-7.

10. Isern D, Moreno A (2008) Computer-based execution of clinical guidelines: a review. International journal of medical informatics, vol. 77 , no. 12, pp. 787-808.

11. Hripcsak G, Ludemann P, Pryor T, Wigertz O, Clayton P (1994) Rationale for the Arden Syntax. Computers and biomedical research an international journal, vol. 27 , no. 4, pp. 291-324.

12. Ohno-Machado L. et al. (1998) The guideline interchange format. Journal of the American Medical Informatics Association, vol. 5, no. 4, p. 357.

13. Vier E, Fox J, Johns N, Lyons C, Rahmanzadeh A, Wilson P (1997) PROforma: systems. Computer Methods and Programs in Biomedicine, vol. 2607, no. 97.

14. Tu S et al. (2007) The SAGE Guideline Model: achievements and overview. Journal of the American Medical Informatics Association, vol. 14, no. 5. pp. 589-98.

15. Bottrighi A, Terenziani P, Montani S, Torchio M, Molino G (2006) Clinical guidelines contextualization in GLARE. AMIA Annual Symposium proceedings AMIA Symposium AMIA Symposium, vol. 2006, pp. 860.

16. Geary D, Horstmann C (2007) Java Server Pages documentation. Prentice Hall.

17. US National Library Of Medicine (2011) Unified Medical Language System (UMLS).

18. Oliveira T, Neves J, Costa A, Novais P, Neves J (2012) An Interpretable Guideline Model to Handle Incomplete Information. In Omatu S, Santana J, Gonzlez S, Molina J, Bernardos A, Corchado E. (eds) Distributed Computing and Artificial Intelligence - 9th International Conference (DCAI 2012), vol. 151, pp. 437-444.

19. Novais P, Salazar M, Ribeiro J, Analide C, Neves J (2010) Decision Making and Quality-of-Information. In Corchado E, Novais P, Analide C, Sedano J (eds) Soft Computing Models in Industrial and Environmental Applications, 5th International Workshop (SOCO 2010),Springer - Series Advances in Intelligent and Soft Computing, vol. 73, pp. 187-195.

20. Neves J, Ribeiro J, Pereira P, Alves V, Machado J, Abelha A, Novais P, Analide C, Santos M, Fernndez-Delgado M. (2012) Evolutionary intelligence in asphalt pavement modeling and quality-of-information. Progress In Artificial Intelligence, Springer, vol. 1, issue 1, pp. 119-135. 